\title{
ON LEGENDRE CURVES IN CONTACT PSEUDO-HERMITIAN 3-MANIFOLDS
}

\author{
JI-EUN LEE
}

(Received 28 April 2009)

\begin{abstract}
We find necessary and sufficient conditions for a Legendre curve in a Sasakian manifold to have: (i) a pseudo-Hermitian parallel mean curvature vector field; (ii) a pseudo-Hermitian proper mean curvature vector field in the normal bundle.
\end{abstract}

2000 Mathematics subject classification: primary 58E20.

Keywords and phrases: Legendre curves, contact pseudo-Hermitian CR-manifold, pseudo-Hermitian minimal, pseudo-Hermitian mean curvature vector field.

\section{Introduction}

Given a contact structure $\eta$, we have two compatible structures. One is a Riemannian structure (or metric) $g$, and then we call $(M ; \eta, g)$ a contact Riemannian manifold. The other is an almost CR-structure $(\eta, L)$, where $L$ is the Levi form associated with an endomorphism $J$ on $D$ such that $J^{2}=-I$. In particular, if $J$ is integrable, then we call it the (integrable) CR-structure. The associated almost CR-structure is said to be pseudo-Hermitian, strongly pseudo-convex if the Levi form is Hermitian and positive definite. We call such a manifold a contact strongly pseudo-convex pseudo-Hermitian (or almost CR-) manifold. There is a one-to-one correspondence between the two associated structures given by the relation

$$
g=L+\eta \otimes \eta
$$

where we denote by the same letter $L$ the natural extension of the Levi form to a $(0,2)$ tensor field on $M$. From this point of view, we have two geometries for a given contact structure, that is, one is formed by the Levi-Civita connection $\nabla$, the other is derived by the Tanaka-Webster connection $\hat{\nabla}$ (or the pseudo-Hermitian connection), which is a canonical affine connection on a strongly pseudo-convex CR-manifold.

This work was supported by a National Research Foundation of Korea Grant funded by the Korean Government [NRF-2009-351-C00008].

(C) 2009 Australian Mathematical Publishing Association Inc. 0004-9727/2009 \$16.00 
In the present paper, we study the contact pseudo-Hermitian geometry in the three-dimensional contact Riemannian manifold with respect to the Tanaka-Webster connection $\hat{\nabla}$. Corresponding to the Laplacian mean curvature vector with respect to the Levi-Civita connection $\nabla$ (see $[3,4,7]$ ), we investigate the following for the Tanaka-Webster connection $\hat{\nabla}$ :

$$
\begin{aligned}
\hat{\Delta} \hat{H} & =\lambda \hat{H}, \\
\hat{\triangle}^{\perp} \hat{H} & =\lambda \hat{H},
\end{aligned}
$$

where $\lambda$ is a function, $\hat{H}$ is the pseudo-Hermitian mean curvature vector and $\hat{\nabla}^{\perp}$ denotes the normal connection in the normal bundle.

A curve $\gamma$ satisfying the first equation in the three-dimensional contact Riemannian manifold $M$ is called a curve with pseudo-Hermitian proper mean curvature vector field. A curve $\gamma$ satisfying the second equation in the three-dimensional contact Riemannian manifold $M$ is called a curve with pseudo-Hermitian proper mean curvature vector field in the normal bundle. In Section 3.1 we study Legendre curves with pseudo-Hermitian minimal and parallel pseudo-Hermitian mean curvature vector in Sasakian manifolds. In Section 3.2 we find necessary and sufficient conditions for a Legendre curve with pseudo-Hermitian harmonic mean curvature vector field and proper pseudo-Hermitian mean curvature vector field in Sasakian manifolds. In Section 3.3 we briefly study curves of $A W(k)$ type from the viewpoint of pseudoHermitian geometry.

\section{Preliminaries}

2.1. Contact Riemannian manifolds. A three-dimensional smooth manifold $M^{3}$ is called a contact manifold if it admits a global 1-form $\eta$ such that $\eta \wedge d \eta \neq 0$ everywhere on $M$. This 1 -form $\eta$ is called the contact form on $M$.

Given a contact form $\eta$, we have a unique vector field $\xi$, which is called the characteristic vector field of $(M, \eta)$, satisfying $\eta(\xi)=1$ and $d \eta(\xi, X)=0$ for any vector field $X$.

A Riemannian metric $g$ on $M$ is said to be an associated metric to a contact structure $\eta$ if there exists an endomorphism field $\varphi$ satisfying

$$
\eta(X)=g(X, \xi), \quad d \eta(X, Y)=g(X, \varphi Y), \quad \varphi^{2} X=-X+\eta(X) \xi,
$$

where $X$ and $Y$ are vector fields on $M$. From (2.1), it follows that

$$
\varphi \xi=0, \quad \eta \circ \varphi=0, \quad g(\varphi X, \varphi Y)=g(X, Y)-\eta(X) \eta(Y) .
$$

A Riemannian manifold $M$ equipped with the structure tensors $(\eta, \xi, \varphi, g)$ satisfying (2.1) is said to be a contact Riemannian manifold. We denote it by $M=(M, \eta ; \xi, \varphi, g)$. Given a contact Riemannian manifold $M$, we define an endomorphism field $\mathrm{h}$ by $\mathrm{h}=\frac{1}{2} L_{\xi} \varphi$, where $L_{\xi}$ denotes Lie differentiation in the characteristic direction $\xi$. The endomorphism field $\mathrm{h}$ is called the structural operator of $(M, \eta ; \varphi, \xi, g)$. 
Then we may observe that $h$ is symmetric and satisfies

$$
\begin{gathered}
\mathrm{h} \xi=0, \quad \mathrm{~h} \varphi=-\varphi \mathrm{h}, \\
\nabla_{X} \xi=-\varphi X-\varphi \mathrm{h} X,
\end{gathered}
$$

where $\nabla$ is the Levi-Civita connection of $(M, g)$.

For a three-dimensional contact Riemannian manifold $M^{3}$, one may define naturally an almost complex structure $J$ on $M \times \mathbb{R}$ by

$$
J\left(X, f \frac{d}{d t}\right)=\left(\varphi X-f \xi, \eta(X) \frac{d}{d t}\right),
$$

where $X$ is a vector field tangent to $M, t$ the coordinate of $\mathbb{R}$ and $f$ a function on $M \times \mathbb{R}$. If the almost complex structure $J$ is integrable, then the contact Riemannian manifold $M$ is said to be a Sasakian manifold.

Proposition 2.1. Let $\left(M^{3}, \eta ; \xi, \varphi, g\right)$ be a contact Riemannian 3-manifold. Then the following three conditions are mutually equivalent.

(1) The characteristic vector field $\xi$ is a Killing vector field, that is, $\nabla \xi=-\varphi$.

(2) $\mathrm{h}=0$.

(3) $M$ is Sasakian.

On a Sasakian 3-manifold, the covariant derivative $\nabla \varphi$ is given by

$$
\left(\nabla_{X} \varphi\right) Y=g(X, Y) \xi-\eta(Y) X, \quad X, Y \in \mathfrak{X}(M) .
$$

Let $(T, N, B)$ be the Frenet frame field along $\gamma$. Then the Frenet frame satisfies the following Frenet-Serret equations:

$$
\left\{\begin{array}{l}
\nabla_{T} T= \\
\nabla_{T} N=-\kappa T \quad \kappa N, \\
\nabla_{T} B=
\end{array} \quad+\tau B,\right.
$$

where $\kappa=|\mathcal{T}(\gamma)|=\left|\nabla_{T} T\right|$ is the geodesic curvature of $\gamma$ and $\tau$ its geodesic torsion.

2.2. Pseudo-Hermitian structure and Tanaka-Webster connection. For a threedimensional contact Riemannian manifold $M=\left(M^{3}, \eta ; \xi, \varphi, g\right)$, the tangent space $T_{p} M$ of $M$ at a point $p \in M$ can be decomposed as

$$
T_{p} M=D_{p} \oplus \mathbb{R} \xi_{p}, \quad D_{p}=\left\{v \in T_{p} M \mid \eta(v)=0\right\}
$$

as the direct sum of linear subspaces. Then $D: p \longmapsto D_{p}$ defines a two-dimensional distribution orthogonal to $\xi$, which is called the contact distribution. We see that the restriction $J=\left.\varphi\right|_{D}$ of $\varphi$ to $D$ defines an almost complex structure on $D$. Then the associated almost CR-structure of the contact Riemannian manifold $M$ is given by the holomorphic subbundle

$$
\mathcal{H}=\{X-i J X \mid X \in D\}
$$


of the complexified tangent bundle $T M^{\mathbb{C}}$. Then we see that each fiber $\mathcal{H}_{p}$ is of complex dimension $1, \mathcal{H} \cap \overline{\mathcal{H}}=\{0\}$, and $D \otimes \mathbb{C}=\mathcal{H} \oplus \overline{\mathcal{H}}$. Furthermore, the associated almost CR-structure is always integrable, that is, the space $\Gamma(\mathcal{H})$ of all smooth sections of $\mathcal{H}$ satisfies the integrability condition:

$$
[\Gamma(\mathcal{H}), \Gamma(\mathcal{H})] \subset \Gamma(\mathcal{H})
$$

For $\mathcal{H}$ we define the Levi form $L$ by

$$
L: \Gamma(D) \times \Gamma(D) \rightarrow \mathfrak{F}(M), \quad L(X, Y)=-d \eta(X, J Y),
$$

where $\mathfrak{F}(M)$ denotes the algebra of smooth functions on $M$. Then we see that the Levi form is Hermitian and positive definite. We call the pair $(\eta, L)$ a contact strongly pseudo-convex pseudo-Hermitian structure on $M$.

Now, we recall the Tanaka-Webster connection $[8,10]$ on a contact strongly pseudo-convex pseudo-Hermitian manifold $M=(M, \eta, L)$ with the associated contact Riemannian structure $(\eta, \xi, \varphi, g)$. The Tanaka-Webster connection $\hat{\nabla}$ is defined by

$$
\hat{\nabla}_{X} Y=\nabla_{X} Y+\eta(X) \varphi Y+\left(\nabla_{X} \eta\right)(Y) \xi-\eta(Y) \nabla_{X} \xi
$$

for all vector fields $X, Y$ on $M$. Together with (2.2), $\hat{\nabla}$ may be rewritten as

$$
\hat{\nabla}_{X} Y=\nabla_{X} Y+A(X, Y)
$$

where we have put

$$
A(X, Y)=\eta(X) \varphi Y+\eta(Y)(\varphi X+\varphi \mathrm{h} X)-g(\varphi X+\varphi \mathrm{h} X, Y) \xi .
$$

We see that the Tanaka-Webster connection $\hat{\nabla}$ has the torsion

$$
\hat{T}(X, Y)=2 g(X, \varphi Y) \xi+\eta(Y) \varphi \mathrm{h} X-\eta(X) \varphi \mathrm{h} Y .
$$

In particular, for Sasakian manifolds, (2.6) and the above equation are reduced to:

$$
\begin{gathered}
A(X, Y)=\eta(X) \varphi Y+\eta(Y) \varphi X-g(\varphi X, Y) \xi, \\
\hat{T}(X, Y)=2 g(X, \varphi Y) \xi .
\end{gathered}
$$

Furthermore, the following proposition was proved in [9].

Proposition 2.2. The Tanaka-Webster connection $\hat{\nabla}$ on a three-dimensional contact Riemannian manifold $M=\left(M^{3} ; \eta, \varphi, \xi, g\right)$ is the unique linear connection satisfying the following conditions:

(1) $\hat{\nabla} \eta=0, \hat{\nabla} \xi=0$;

(2) $\hat{\nabla} g=0, \hat{\nabla} \varphi=0$;

(3) $\hat{T}(X, Y)=-\eta([X, Y]) \xi, X, Y \in \Gamma(D)$;

(4) $\hat{T}(\xi, \varphi Y)=-\varphi \hat{T}(\xi, Y), Y \in \Gamma(D)$. 


\section{Legendre curves in pseudo-Hermitian geometry}

Let $\gamma: I \rightarrow M^{3}$ be a curve parameterized by arc-length in a contact Riemannian 3-manifold $M^{3}$. We may define a Frenet frame field $(T, N, B)$ along $\gamma$ with respect to the Tanaka-Webster connection $\hat{\nabla}$. This satisfies the following Frenet-Serret equations for $\hat{\nabla}$ :

$$
\begin{cases}\hat{\nabla}_{T} T= & \hat{\kappa} N \\ \hat{\nabla}_{T} N=-\hat{\kappa} T & \quad+\hat{\tau} B \\ \hat{\nabla}_{T} B= & -\hat{\tau} N\end{cases}
$$

where $\hat{\kappa}=\left|\hat{\nabla}_{T} T\right|$ is the pseudo-Hermitian curvature of $\gamma$ and $\hat{\tau}$ its pseudo-Hermitian torsion. A pseudo-Hermitian helix is a curve whose pseudo-Hermitian curvature and pseudo-Hermitian torsion are constants. In particular, curves with constant nonzero pseudo-Hermitian curvature and zero pseudo-Hermitian torsion are called pseudoHermitian circles. Note that pseudo-Hermitian geodesics are regarded as pseudoHermitian helices whose pseudo-Hermitian curvature and pseudo-Hermitian torsion are zero.

Blair and Baikoussis introduced the notion of Legendre curves in a contact Riemannian manifold. A one-dimensional integral submanifold in the contact subbundle is called a Legendre curve (see [2]).

3.1. Parallel pseudo-Hermitian mean curvature vector. The pseudo-Hermitian mean curvature vector field $\hat{H}$ of a curve $\gamma$ in three-dimensional contact Riemannian manifolds is defined by

$$
\hat{H}=\hat{\nabla}_{\dot{\gamma}} \dot{\gamma}=\hat{\kappa} N .
$$

In particular, for a Legendre curve $\gamma$ we get

$$
\hat{H}=\hat{\nabla}_{\dot{\gamma}} \dot{\gamma}=\hat{\kappa} \varphi \dot{\gamma} .
$$

Differentiating $\varphi \dot{\gamma}$ along $\gamma$ for $\hat{\nabla}$, we get $\hat{\tau}=0$ and this proves the following proposition.

PROPOSITION $3.1[5,6]$. If a nongeodesic curve in a three-dimensional contact Riemannian manifold for Tanaka-Webster connection $\hat{\nabla}$ is a Legendre curve, then $\hat{\tau}=0$.

For a curve $\gamma$ in a three-dimensional Sasakian manifold $M$, from (2.5) and (2.7) we get

$$
\hat{\nabla}_{\dot{\gamma}} \dot{\gamma}=\nabla_{\dot{\gamma}} \dot{\gamma}+2 \eta(\dot{\gamma}) \varphi \dot{\gamma}
$$

and for a Legendre curve $\gamma$ in a three-dimensional Sasakian manifold $M$ we can see that $\hat{\nabla}_{\dot{\gamma}} \dot{\gamma}=\nabla_{\dot{\gamma}} \dot{\gamma}$. So we have the following proposition.

PROPOSITION 3.2 [5, 6]. For a Legendre curve $\gamma$ in a three-dimensional Sasakian manifold $M, \gamma$ is pseudo-Hermitian minimal if and only if it is minimal. 
First, we consider Legendre curves $\gamma$ in a three-dimensional contact Riemannian manifold with respect to the Levi-Civita connection $\nabla$. If we define a parallel mean curvature vector field by $\nabla \frac{\perp}{\dot{\gamma}} H=0$, then we get the following lemma.

LEMMA 3.3 [7]. For Legendre curves $\gamma$ in three-dimensional Sasakian manifolds (with respect to the Levi-Civita connection $\nabla), \gamma$ has a parallel mean curvature vector field if and only if it is minimal.

PROOF. Using the Frenet-Serret equation for the Levi-Civita connection $\nabla$,

$$
\nabla_{\dot{\gamma}} H=\nabla_{\dot{\gamma}} \nabla_{\dot{\gamma}} \dot{\gamma}=-\kappa^{2} \dot{\gamma}+\kappa^{\prime} \varphi \dot{\gamma}+\kappa \xi
$$

Since $\gamma$ has a parallel mean curvature vector field, we can see that $\kappa=0$. The converse is straightforward.

In pseudo-Hermitian geometry, we investigate the following definition.

DEFINITION 3.4. In three-dimensional contact Riemannian manifolds $M^{3}$ with respect to the Tanaka-Webster connection $\hat{\nabla}$, a vector field $X$ normal to curve $\gamma$ is said to be pseudo-Hermitian parallel if $\hat{\nabla} \underset{\dot{\gamma}}{\perp} X=0$.

On differentiating (3.2),

$$
\hat{\nabla}_{\dot{\gamma}} \hat{H}=-\hat{\kappa}^{2} \dot{\gamma}+\hat{\kappa}^{\prime} \varphi \dot{\gamma}
$$

Using (3.4) and Definition 3.4, we get that $\hat{\kappa}$ is a constant. Thus, from Proposition 3.1, we can see that $\hat{\tau}=0$ for a Legendre curve in a three-dimensional contact Riemannian manifold. So we obtain the following theorem.

THEOREM 3.5. For a Legendre curve $\gamma$ in a three-dimensional Sasakian manifold $M$, $\gamma$ is a curve with pseudo-Hermitian parallel mean curvature vector if and only if $\gamma$ is a pseudo-Hermitian circle.

3.2. Proper pseudo-Hermitian mean curvature vector field. For a curve $\gamma$ in a three-dimensional contact Riemannian manifold with respect to the Tanaka-Webster connection $\hat{\nabla}$,

$$
\hat{\Delta} \hat{H}=-\hat{\nabla}_{\dot{\gamma}} \hat{\nabla}_{\dot{\gamma}} \hat{\nabla}_{\dot{\gamma}} \dot{\gamma}
$$

where $\hat{H}$ is the pseudo-Hermitian mean curvature vector. Moreover, the Laplacian of the pseudo-Hermitian mean curvature vector in the normal bundle is defined by

$$
\hat{\Delta}^{\perp} \hat{H}=-\hat{\nabla}_{\dot{\gamma}}^{\perp} \hat{\nabla}_{\dot{\gamma}}^{\perp} \hat{\nabla}_{\dot{\gamma}}^{\perp} \dot{\gamma}
$$

where $\hat{\nabla}^{\perp}$ denotes the normal connection in the normal bundle.

A curve $\gamma$ in three-dimensional contact Riemannian manifold $M$ is called a curve with pseudo-Hermitian proper mean curvature vector field if $\hat{\Delta} \hat{H}=\lambda \hat{H}$, where $\lambda$ is a function. In particular, if $\hat{\Delta} \hat{H}=0$ then it reduces to a curve with pseudo-Hermitian harmonic mean curvature vector field. 
A curve $\gamma$ is called a curve with pseudo-Hermitian proper mean curvature vector field in the normal bundle if $\hat{\Delta}^{\perp} \hat{H}=\lambda \hat{H}$, where $\hat{\Delta}^{\perp} \hat{H}$ is the Laplacian of the pseudoHermitian mean curvature vector in the normal bundle and $\lambda$ is a function. In particular, if $\hat{\Delta}^{\perp} \hat{H}=0$ then it reduces to a curve with pseudo-Hermitian harmonic mean curvature vector field in the normal bundle (see [7]).

Using (3.1), we have the following lemma.

LEMMA 3.6. Let $\gamma$ be a Legendre curve in a three-dimensional Sasakian manifold $M$. Then

$$
\begin{gathered}
\hat{\nabla}_{\dot{\gamma}} \hat{\nabla}_{\dot{\gamma}} \hat{\nabla}_{\dot{\gamma}} \dot{\gamma}=-3 \hat{\kappa} \hat{\kappa}^{\prime} \dot{\gamma}+\left(\hat{\kappa}^{\prime \prime}-\hat{\kappa}^{3}\right) \varphi \dot{\gamma}, \\
\hat{\nabla}_{\dot{\gamma}}^{+} \hat{\nabla}_{\dot{\gamma}}^{+} \hat{\nabla}_{\dot{\gamma}}^{\perp} \dot{\gamma}=\hat{\kappa}^{\prime \prime} \varphi \dot{\gamma} .
\end{gathered}
$$

First, we study the pseudo-Hermitian mean curvature vector field.

THEOREM 3.7. Let $\gamma$ be a Legendre curve in a three-dimensional Sasakian manifold $M$. Then $\gamma$ has pseudo-Hermitian proper mean curvature vector field if and only if $\gamma$ is a minimal or pseudo-Hermitian circle satisfying $\hat{\kappa}^{2}=\lambda$ for nonzero constant $\hat{\kappa}$.

Proof. From (3.5), the condition $\hat{\Delta} \hat{H}=\lambda \hat{H}$ gives

$$
3 \hat{\kappa} \hat{\kappa}^{\prime} \dot{\gamma}-\left(\hat{\kappa}^{\prime \prime}-\hat{\kappa}^{3}\right) \varphi \dot{\gamma}=\lambda \hat{\kappa} \varphi \dot{\gamma}
$$

which implies that $\hat{\kappa}=0$ or $\hat{\kappa}^{2}-\lambda=0$ for a nonzero constant $\hat{\kappa}$. The converse follows easily.

In particular, for the case of $\lambda=0$ we have the following corollary.

Corollary 3.8. Let $\gamma$ be a Legendre curve in a three-dimensional Sasakian manifold $M$. Then $\hat{\Delta} \hat{H}=0$ if and only if $\gamma$ is minimal.

Next, we study pseudo-Hermitian mean curvature vector fields in the normal bundle.

THEOREM 3.9. Let $\gamma$ be a Legendre curve in a three-dimensional Sasakian manifold $M$ and suppose that $\lambda$ is a nonzero constant. Then $\gamma$ has a pseudoHermitian proper mean curvature vector field in the normal bundle if and only if $\hat{\kappa}(s)=\cos ( \pm \sqrt{\lambda} s+c)$, where $c$ is a constant.

PROOF. In view of (3.6), the condition $\hat{\Delta}^{\perp} \hat{H}=\lambda \hat{H}$ gives

$$
-\hat{\kappa}^{\prime \prime} \varphi \dot{\gamma}=\lambda \hat{\kappa} \varphi \dot{\gamma}
$$

which implies that $\kappa^{\prime \prime}+\lambda \hat{\kappa}=0$. Since $\lambda$ is a nonzero constant, we find that $\hat{\kappa}(s)=$ $\cos ( \pm \sqrt{\lambda} s+c)$, where $c$ is a constant. The converse is straightforward. 
For the case of $\lambda=0$, we have the following corollary.

COROLlary 3.10. Let $\gamma$ be a Legendre curve in a three-dimensional Sasakian manifold $M$. Then $\hat{\Delta}^{\perp} \hat{H}=0$ if and only if $\hat{\kappa}(s)=a s+b$, where a and $b$ are constants.

Proof. From (3.6), the condition $\hat{\Delta}^{\perp} \hat{H}=0$ gives

$$
\kappa^{\prime \prime}(s)=0,
$$

which implies that $\hat{\kappa}(s)=a s+b$, where $a$ and $b$ are constants. The converse follows easily.

Next, from the study of the Levi-Civita connection $\nabla$, we can see that pseudoHermitian geometry is different from Riemmanian geometry. For Legendre curves $\gamma$ in three-dimensional contact Riemannian manifolds with respect to the Levi-Civita connection $\nabla$, if we define a harmonic mean curvature vector field by $\Delta^{\perp} H=0$, then we get the following lemma.

LEMMA 3.11 [7]. For Legendre curves $\gamma$ in three-dimensional Sasakian manifolds (with respect to the Levi-Civita connection $\nabla$ ), $\gamma$ has harmonic a mean curvature vector field if and only if it is minimal.

PROOF. Using the Frenet-Serret equation for the Levi-Civita connection $\nabla$,

$$
\Delta^{\perp} H=\nabla_{\dot{\gamma}}^{\perp} \nabla_{\dot{\gamma}}^{\perp} H=\left(\kappa^{\prime \prime}+\kappa^{\prime}-\kappa\right) \varphi \dot{\gamma}+2 \kappa^{\prime} \xi .
$$

Since $\gamma$ has harmonic mean curvature vector field, we can see that $\kappa=0$.

3.3. On curves of $\boldsymbol{A} \boldsymbol{W}(\boldsymbol{k})$ type. In [1] Arslan and Ozgur studied curves of $A W(k)$ type. In this section, we investigate curves of $A W(k)$ type from the viewpoint of pseudo-Hermitian geometry and we find necessary and sufficient conditions for them.

DEFINITION 3.12. Let $M$ be a three-dimensional contact Riemannian manifold with respect to the Tanaka-Webster connection $\hat{\nabla}$. Curves are of pseudo-Hermitian $A W(1)$ type if they satisfy

$$
\left(\hat{\nabla}_{\dot{\gamma}} \hat{\nabla}_{\dot{\gamma}} \hat{\nabla}_{\dot{\gamma}} \dot{\gamma}\right)^{\perp}=0
$$

of pseudo-Hermitian $A W(2)$ type if they satisfy

$$
\left(\hat{\nabla}_{\dot{\gamma}} \hat{\nabla}_{\dot{\gamma}} \hat{\nabla}_{\dot{\gamma}} \dot{\gamma}\right)^{\perp} \wedge\left(\hat{\nabla}_{\dot{\gamma}} \hat{\nabla}_{\dot{\gamma}} \dot{\gamma}\right)^{\perp}=0 \text {, }
$$

and of pseudo-Hermitian $A W(3)$ type if they satisfy

$$
\left(\hat{\nabla}_{\dot{\gamma}} \hat{\nabla}_{\dot{\gamma}} \hat{\nabla}_{\dot{\gamma}} \dot{\gamma}\right)^{\perp} \wedge\left(\hat{\nabla}_{\dot{\gamma}} \dot{\gamma}\right)^{\perp}=0
$$

LEMMA 3.13. Let $\gamma$ be a Legendre curve in a three-dimensional Sasakian manifold $M$. Then we have following.

(i) $\gamma$ is of the pseudo-Hermitian $A W(1)$ type if and only if $\hat{\kappa}(s)= \pm \sqrt{2} /(s+c)$, where $c$ is a constant. 
(ii) For a function $\hat{\kappa}, \gamma$ always satisfies the condition for the pseudo-Hermitian $A W(2)$ or $A W(3)$ type.

In [7] Ozgur and Tripathi studied Legendre curves of $A W(k)$ type in Sasakian manifolds for the Levi-Civita connection in detail.

\section{References}

[1] K. Arslan and C. Ozgur, 'Curves and surfaces of $A W(k)$ type', in: Geometry and Topology of Submanifolds, IX (Valenciennes/Lyon/Leuven, 1997) (World Scientific, River Edge, NJ, 1999), pp. 21-26.

[2] C. Baikoussis and D. E. Blair, 'On Legendre curves in contact 3-manifolds', Geom. Dedicata 49 (1994), 135-142.

[3] M. Barros and O. J. Garay, 'On submanifolds with harmonic mean curvature', Proc. Amer. Math. Soc. 123(8) (1995), 2545-2549.

[4] B.-Y. Chen, Total Mean Curvature and Submanifolds of Finite Type, Series in Pure Mathematics, 1 (World Scientific, Singapore, 1984).

[5] J. T. Cho, J. Inoguchi and J.-E. Lee, 'Affine biharmonic submanifolds in three-dimensional pseudoHermitian geometry', Abh. Math. Sem. Univ. Hamburg. 79 (2009), 113-133.

[6] J. T. Cho and J.-E. Lee, 'Slant curves in contact pseudo-Hermitian 3-manifolds', Bull. Aust. Math. Soc. 78(3) (2008), 383-396.

[7] C. Ozgur and M. M. Tripathi, 'On Legendre curves in $\alpha$-Sasakian manifolds', Bull. Malays. Math. Sci. Soc. (2) 31(1) (2008), 91-96.

[8] N. Tanaka, 'On non-degenerate real hypersurfaces, graded Lie algebras and Cartan connections', Japan. J. Math. 2 (1976), 131-190.

[9] S. Tanno, 'Variational problems on contact Riemannian manifolds', Trans. Amer. Math. Soc. 314 (1989), 349-379.

[10] S. M. Webster, 'Pseudohermitian structures on a real hypersurface', J. Differential Geom. 13 (1978), 25-41.

JI-EUN LEE, Department of Mathematics, Kyungpook National University,

Taegu, 702-701, Korea

e-mail: jieunlee12@gmail.com 